\title{
Research Article \\ CURRENT TRENDS IN OILSEED CROPS PRODUCTION: AN OVERVIEW
}

\section{SHARMA AMOD}

Department of Agricultural Economics, SASRD, Nagaland University, Medziphema Campus, Dimapur, Nagaland, 797106

*Corresponding Author: Email-hodaec_sasrd@yahoo.co.in

Received: January 27, 2018; Revised: February 05, 2018; Accepted: February 06, 2018; Published: February 15, 2018

\begin{abstract}
In this study, current trend in oilseed crops production has been emphasised, the study was based on secondary data from the year 1950-51 to 2012-13. The data is based on several governmental documents and web-sites. The linear, quadratic and exponential functions were fitted in order to analyse the trend in area, production and productivity of oilseed crops in India. Quadratic functional form was employed to fit the trend due to its higher $\mathrm{R}^{2}$ value as compared to other two forms. Besides these, compound growth rate, coefficient of variation (CV) and instability index are also computed. Similarly, the effects of area, productivity and their interactions towards increasing production were also estimated in the present study. The results of the study revealed that the ' $c$ ' value in the quadratic functional forms for area, production and productivity were positive and significant for the total oilseed crops in the country more particularly during Phase I, Phase II, Phase III, Phase IV, Phase V, Phase VI, Phase VII and overall. The growing of oilseed crops was moderate risky as revealed by the lower CV. The CV of area, production and productivity of oilseed crops were less than 43.68 per cent. The instability indices for area, production and productivity for oilseed crops were positive and thereby indicating less risk for growing oilseed crops in coming days. The increase in production is due to increase in area as well as interaction of area and productivity of oilseed crops in the study periods.
\end{abstract}

Keywords- Trend, area, production, productivity, oilseed, crops.

Citation: Sharma Amod (2018) Current Trends in Oilseed Crops Production: An Overview. International Journal of Agriculture Sciences, ISSN: 0975-3710 \& E-ISSN: 0975-9107, Volume 10, Issue 3, pp.-5104-5114. DOl: http://dx.doi.org/10.9735/0975-3710.10.3.5104-5114

Copyright: Copyright@2018 Sharma Amod. This is an open-access article distributed under the terms of the Creative Commons Attribution License, which permits unrestricted use, distribution and reproduction in any medium, provided the original author and source are credited.

Academic Editor / Reviewer: Dr Anil Kumar, Dr Krishnamoorthy Ramasamy, AS Gawali

\section{Introduction}

The demand and supply for oilseed crops was out strips, the production and the gap were increasing over the time. The total oilseed production increased from 4.73 lakh tonnes to 31.10 lakh tones, while yield increased from $419 \mathrm{~kg} / \mathrm{ha}$ to $1159 \mathrm{~kg} /$ ha during the period from 1950-51 to 2012-13 [1-3]. As the Country has enjoys the abundance of natural resources viz; flora and fauna has ever importance of rich natural resources has been neglected in the past. This has hampered the development process in tune since it is difficult to utilize these natural resources for the benefit of the people. If the natural resources are not well recognised and managed.

Now, there is a need to formulate appropriate strategies to boost the historical significance before formulating the oil production in the right direction, but it will be wise to understand any strategies for the development and one must identify the existing trends in area, production and productivity that play an important role in the development process $([8,15,18]$. It is desire that there should clean picture of demand and supply gap by examining the trend as well as the required quantity to meet the future consumption requirement with the increasing population with available resources. In this study, an attempt has been made to analysis the trends of area, production and productivity of different oilseed crops in the Country. The specific objectives of the study are:

(i) To study the growth rates in area, production and yield of different oilseed crops in India; and

(ii) To find out the variability in area, production and yield of different oilseed crops with respect to groundnut, mustard and rapeseed, soybean and sunflower in
India.

\section{Data Base and Research Methodology}

The present study has been conducted entirely based on secondary data. The secondary data in relation to area, production and productivity of different oilseed crops from 1950-51 to 2012-13 were collected from different government sources [1-3]. However, data for three years (2010-11 to 2012-13) were forecasted based on previous year trends and generated for the present study $[10,16]$. The study entails temporal as well as spatial analyses for estimation of growth in area, production and productivity of oilseed crops.

To analyse the trend in area, production and productivity of different oilseed crops, the following different functional forms were fitted.

(1) Linear function $\quad Y=a+b x$

(2) Quadratic function $Y=a+b x+c x^{2}$

(3) Exponential function $Y=a \cdot b^{x}$

Where, $Y=$ Area, production and productivity of different oilseed crops $\mathrm{x}=$ Time variable

From the raw data on area, production and productivity of different oilseed crops after analyzed the different parameters / form of function (i.e.; Linear, Quadratic and Exponential type) it was decided based on the highest co-efficient of determination $\left(R^{2}\right)$ to calculate the trend as suitable model.

As Exponential function model was found to be a good model due to the better results as compared to remaining two models; so, data were computed for area, 
production and productivity to work out the compound Growth Rates with the following method:

$$
\text { Exponential trend equation: } Y=a \cdot b^{x} \text {. }
$$

Where, $\mathrm{x}$ is the time variable, $\mathrm{y}$ is the variable for which growth rate is calculated and $b$ is the regression co-efficient of $Y$ on $x$.

$$
\text { Compound Growth percentage (CGR \%) }=(b-1) \times 100
$$

The significance of growth rates was tested by applying student ' 1 ' test where $t=g$ I SE (r), with (N-2) d. f. where $r$ is the growth and $N$ is the total number of years considered under study.

SE $(r)=100 b / 0.4329 \sqrt{ }\left[\left(\sum \log r^{2}\right)-\left(\sum \log Y\right)^{2} / N-(\log b)^{2} \sum x^{2}\right] /(N-2) \sum x 2$ To understand the variation of magnitude in the selected data of area, production and productivity into the seven sub period as well as overall time period the coefficient of variation was calculated. Therefore, Instability Index in the percentage was calculated over the selected period with the help of following formula given as under:

$$
\text { Instability Index }(I)=\left(I-R^{2}\right) \times C V^{2}
$$

The effect of area, productivity and their interaction towards increasing production were worked out by using the following formula. Similar technique were adopted / employed by Cavery (1991)[4]; Das and Sharma (2012)[5]; Dhakre \& Sharma (2010a)[6]; Dhakre and Sharma (2010b)[7]; Sharma (2012)[8]; Sharma (2013a)[9]; Sharma (2014)[11]; Sharma (2015a)[12]; Sharma (2015b)[13]; Sharma (2015c)[14]; Sharma \& Kalita (2004)[15]; Sharma \& Kalita (2008)[16]; Yadav et. al., (2017) [17].

$$
\Delta P=Y_{0} \Delta A+A_{0} \Delta Y+\Delta A \Delta Y
$$

Where, $\Delta A=A_{n}-A_{0}$

$\Delta Y=Y_{n}-Y_{0}$

$\Delta P=A_{n}-A_{0}$

$A_{0}, P_{0}$ and $Y_{0}$ represent the area, production and productivity in the base year and $A_{n}, P_{n}$ and $Y_{n}$ the corresponding area, production and productivity in the current year. The first, second and third on the right side of above equation represent area, productivity and interaction effect, respectively.

The periods from 1950-51 to 2012-13 was divided into seven sub-periods viz.; (i) 1950-51 to 1959-60; (ii) 1960-61 to 1969-70; (iii) 1970-71 to 1979-80; (iv) 1980-81 to 1989-90; (v) 1990-91 to 1999-00; (vi) 2000-01 to 2009-10 and (vii) 2010-11 to

\begin{tabular}{|c|c|c|c|c|}
\hline \multicolumn{5}{|c|}{ Table-1 $R^{2}$ value of linear, quadratic and exponential function for different oilseed crops } \\
\hline Period & Aspects & Linear & Quadratic & Exponential \\
\hline \multicolumn{5}{|c|}{ Groundnut } \\
\hline \multirow{3}{*}{ Period - I } & Area & 0.78495 & 0.81308 & 0.61865 \\
\hline & Production & 0.76935 & 0.75278 & 0.60219 \\
\hline & Productivity & 0.09680 & 0.07313 & 0.07568 \\
\hline \multirow{3}{*}{ Period - II } & Area & 0.27704 & 0.22159 & 0.34724 \\
\hline & Production & 0.00055 & 0.00104 & 0.00078 \\
\hline & Productivity & 0.07215 & 0.06393 & 0.07865 \\
\hline \multirow{3}{*}{ Period - III } & Area & 0.02411 & 0.01630 & 0.03252 \\
\hline & Production & 0.02739 & 0.03046 & 0.02986 \\
\hline & Productivity & 0.04824 & 0.05003 & 0.05044 \\
\hline \multirow{3}{*}{ Period - IV } & Area & 0.34108 & 0.35931 & 0.30977 \\
\hline & Production & 0.28032 & 0.29199 & 0.26202 \\
\hline & Productivity & 0.18378 & 0.18913 & 0.17995 \\
\hline \multirow{3}{*}{ Period - V } & Area & 0.87297 & 0.87560 & 0.86795 \\
\hline & Production & 0.03720 & 0.04307 & 0.05593 \\
\hline & Productivity & 0.09044 & 0.08847 & 0.10560 \\
\hline \multirow{3}{*}{ Period - Vl } & Area & 0.17444 & 0.17904 & 0.17782 \\
\hline & Production & 0.01087 & 0.00984 & 0.00958 \\
\hline & Productivity & 0.05557 & 0.05381 & 0.06043 \\
\hline \multirow{3}{*}{$\begin{array}{c}\text { Period - } \\
\text { VII }\end{array}$} & Area & 0.01312 & 0.01312 & 0.03330 \\
\hline & Production & 0.08955 & 0.08955 & 0.10826 \\
\hline & Productivity & 0.99999 & 0.99999 & 0.99999 \\
\hline \multirow{3}{*}{ Overall } & Area & 0.03589 & 0.00013 & 0.24283 \\
\hline & Production & 0.48258 & 0.37007 & 0.59401 \\
\hline & Productivity & 0.05371 & 0.05104 & 0.17498 \\
\hline \multicolumn{5}{|c|}{ Rapeseed \&Mustard } \\
\hline \multirow{3}{*}{ Period - I } & Area & 0.62623 & 0.62500 & 0.58144 \\
\hline & Production & 0.41729 & 0.41339 & 0.37425 \\
\hline & Productivity & 0.00239 & 0.00000 & 0.01312 \\
\hline \multirow{3}{*}{ Period - II } & Area & 0.01567 & 0.01801 & 0.01341 \\
\hline & Production & 0.07851 & 0.09409 & 0.03332 \\
\hline & Productivity & 0.12158 & 0.14112 & 0.08745 \\
\hline \multirow{3}{*}{ Period - III } & Area & 0.00828 & 0.00872 & 0.00765 \\
\hline & Production & 0.06075 & 0.06883 & 0.05320 \\
\hline & Productivity & 0.08685 & 0.09681 & 0.06552 \\
\hline \multirow{3}{*}{ Period - IV } & Area & 0.34662 & 0.37366 & 0.29661 \\
\hline & Production & 0.75159 & 0.76897 & 0.76414 \\
\hline & Productivity & 0.80348 & 0.80270 & 0.82145 \\
\hline \multirow{3}{*}{ Period - V } & Area & 0.14431 & 0.13590 & 0.15421 \\
\hline & Production & 0.05358 & 0.05142 & 0.05126 \\
\hline & Productivity & 0.00210 & 0.00223 & 0.00014 \\
\hline \multirow{3}{*}{ Period - VI } & Area & 0.26339 & 0.24892 & 0.32550 \\
\hline & Production & 0.40071 & 0.38663 & 0.44723 \\
\hline & Productivity & 0.44105 & 0.43879 & 0.43529 \\
\hline
\end{tabular}
2012-13 and contribution of area, productivity and their interactions to total production were worked out separately for total period and each sub-period.

\section{Results and Discussion}


Sharma Amod

\begin{tabular}{|c|c|c|c|c|}
\hline \multirow{3}{*}{$\begin{array}{c}\text { Period - } \\
\text { VII }\end{array}$} & Area & 0.93823 & 0.93596 & 0.93822 \\
\hline & Production & 0.59626 & 0.59168 & 0.59371 \\
\hline & Productivity & 0.71690 & 0.71270 & 0.71888 \\
\hline \multirow{3}{*}{ Overall } & Area & 0.83973 & 0.80953 & 0.75781 \\
\hline & Production & 0.88245 & 0.89690 & 0.82032 \\
\hline & Productivity & 0.87759 & 0.89853 & 0.66199 \\
\hline \multicolumn{5}{|c|}{ Soybean } \\
\hline \multirow{3}{*}{ Period - I } & Area & 0.78019 & 0.84260 & 0.61306 \\
\hline & Production & 0.05518 & 0.11274 & 0.00120 \\
\hline & Productivity & 0.45472 & 0.39276 & 0.53347 \\
\hline \multirow{3}{*}{ Period - II } & Area & 0.87417 & 0.86161 & 0.86712 \\
\hline & Production & 0.00905 & 0.02073 & 0.00157 \\
\hline & Productivity & 0.85628 & 0.87623 & 0.82774 \\
\hline \multirow{3}{*}{ Period - III } & Area & 0.75517 & 0.79349 & 0.94331 \\
\hline & Production & 0.76851 & 0.80205 & 0.84269 \\
\hline & Productivity & 0.59302 & 0.59920 & 0.62525 \\
\hline \multirow{3}{*}{ Period - IV } & Area & 0.94401 & 0.94945 & 0.92885 \\
\hline & Production & 0.83616 & 0.84918 & 0.88210 \\
\hline & Productivity & 0.05191 & 0.05751 & 0.02713 \\
\hline \multirow{3}{*}{ Period - V } & Area & 0.96804 & 0.96172 & 0.94570 \\
\hline & Production & 0.94319 & 0.94335 & 0.92660 \\
\hline & Productivity & 0.45197 & 0.45958 & 0.42948 \\
\hline \multirow{3}{*}{ Period - VI } & Area & 0.93219 & 0.93909 & 0.92427 \\
\hline & Production & 0.01949 & 0.01721 & 0.14430 \\
\hline & Productivity & 0.00731 & 0.00591 & 0.03312 \\
\hline & Area & 0.12351 & 0.12351 & 0.13552 \\
\hline & Production & 0.41390 & 0.41390 & 0.41097 \\
\hline VII & Productivity & 0.11258 & 0.11259 & 0.10075 \\
\hline & Area & 0.80315 & 0.93908 & 0.68209 \\
\hline Overall & Production & 0.20655 & 0.25450 & 0.67741 \\
\hline & Productivity & 0.21323 & 0.22439 & 0.60736 \\
\hline & & unflower & & \\
\hline & Area & 0.77981 & 0.61686 & 0.91557 \\
\hline Period - I & Production & 0.00230 & 0.02048 & 0.00343 \\
\hline & Productivity & 0.76573 & 0.62583 & 0.88915 \\
\hline & Area & 0.32694 & 0.25805 & 0.42856 \\
\hline Period - II & Production & 0.34327 & 0.39559 & 0.29293 \\
\hline & Productivity & 0.65533 & 0.59566 & 0.69826 \\
\hline & Area & 0.01635 & 0.00724 & 0.00477 \\
\hline Period - III & Production & 0.05491 & 0.03722 & 0.07870 \\
\hline & Productivity & 0.39963 & 0.36498 & 0.57607 \\
\hline & Area & 0.79609 & 0.78298 & 0.80708 \\
\hline Period - IV & Production & 0.75019 & 0.73997 & 0.55514 \\
\hline & Productivity & 0.33112 & 0.31254 & 0.34858 \\
\hline & Area & 0.20294 & 0.22057 & 0.20548 \\
\hline Period - V & Production & 0.18287 & 0.20276 & 0.18183 \\
\hline & Productivity & 0.00012 & 0.00018 & 0.00004 \\
\hline & Area & 0.18574 & 0.16900 & 0.25232 \\
\hline Period - VI & Production & 0.33841 & 0.32095 & 0.40174 \\
\hline & Productivity & 0.16565 & 0.16869 & 0.15914 \\
\hline & Area & 0.37458 & 0.37909 & 0.13565 \\
\hline Period - & Production & 0.26618 & 0.27030 & 0.08335 \\
\hline VII & Productivity & 0.99561 & 0.99621 & 0.99022 \\
\hline & Area & 0.64387 & 0.62014 & 0.57290 \\
\hline Overall & Production & 0.65290 & 0.65473 & 0.39502 \\
\hline & Productivity & 0.30477 & 0.33423 & 0.06088 \\
\hline & & tal Oilseed & & \\
\hline & Area & 0.83000 & 0.84402 & 0.71678 \\
\hline Period - I & Production & 0.75843 & 0.72289 & 0.64567 \\
\hline & Productivity & 0.31969 & 0.27414 & 0.26337 \\
\hline & Area & 0.10635 & 0.07251 & 0.15222 \\
\hline Period - II & Production & 0.01051 & 0.01065 & 0.00771 \\
\hline & Productivity & 0.00076 & 0.00001 & 0.00413 \\
\hline & Area & 0.14555 & 0.14821 & 0.14082 \\
\hline Period - III & Production & 0.03883 & 0.03831 & 0.04021 \\
\hline & Productivity & 0.01060 & 0.01009 & 0.01307 \\
\hline & Area & 0.73744 & 0.76008 & 0.72619 \\
\hline Period - IV & Production & 0.58239 & 0.59769 & 0.58030 \\
\hline & Productivity & 0.43067 & 0.43794 & 0.42737 \\
\hline & Area & 0.02031 & 0.01518 & 0.02631 \\
\hline Period - V & Production & 0.47464 & 0.45817 & 0.50966 \\
\hline & Productivity & 0.57314 & 0.56384 & 0.59158 \\
\hline & Area & 0.57008 & 0.55836 & 0.58834 \\
\hline Period - VI & Production & 0.53115 & 0.52137 & 0.51242 \\
\hline & Productivity & 0.31513 & 0.30989 & 0.32100 \\
\hline
\end{tabular}




\begin{tabular}{|c|c|c|c|c|}
\hline \multirow{3}{*}{ Period - VII } & Area & 0.75345 & 0.75745 & 0.75969 \\
\cline { 2 - 5 } & Production & 0.02376 & 0.02236 & 0.02453 \\
\cline { 2 - 5 } & Productivity & 0.80848 & 0.81213 & 0.76950 \\
\hline \multirow{3}{*}{ Overall } & Area & 0.92071 & 0.87210 & 0.82286 \\
\cline { 2 - 5 } & Production & 0.87178 & 0.92882 & 0.72730 \\
\cline { 2 - 5 } & Productivity & 0.83124 & 0.90445 & 0.58219 \\
\hline
\end{tabular}

Coefficients of determination $\left(\mathrm{R}^{2}\right)$ by fitting linear, quadratic and exponential functions are computed and presented in [Table-1].

[Table-1] shows that the $\mathrm{R}^{2}$ values of quadratic function for area, production and productivity for different oilseed crops in India are higher than linear and exponential functions. Hence, the quadratic functional form was selected for fitting trend of area, production and productivity of different oilseed crops in the Country [8].

Table-2 Results of the fitted trend for different oilseed crops (quadratic function)

\begin{tabular}{|c|c|c|c|c|}
\hline Period & Aspects & a & $b$ & c \\
\hline \multicolumn{5}{|c|}{ Groundnut } \\
\hline \multirow{3}{*}{ Period - I } & Area & 1.00172 & 0.493201 & -0.17286 \\
\hline & Production & 1.872882 & 0.991475 & 0.371256 \\
\hline & Productivity & -0.6916 & -1.07214 & 1.145425 \\
\hline \multirow{3}{*}{ Period - II } & Area & 0.042733 & 0.998382 & -1.01134 \\
\hline & Production & -0.04173 & 1.001294 & 1.013032 \\
\hline & Productivity & 0.043141 & -0.98762 & 0.986392 \\
\hline \multirow{3}{*}{ Period - III } & Area & -0.00763 & 1.001958 & -1.00186 \\
\hline & Production & 0.008377 & 0.997871 & 0.999873 \\
\hline & Productivity & -0.00817 & -0.998 & 1.000094 \\
\hline \multirow{3}{*}{ Period - IV } & Area & 0.008512 & 0.998673 & -0.99987 \\
\hline & Production & -0.00623 & 1.000694 & 1.00125 \\
\hline & Productivity & 0.006089 & -0.99936 & 0.998726 \\
\hline \multirow{3}{*}{ Period - V } & Area & -0.01122 & 1.004318 & -1.00623 \\
\hline & Production & 0.011469 & 0.99559 & 1.001948 \\
\hline & Productivity & -0.01151 & -0.99357 & 0.998017 \\
\hline \multirow{3}{*}{ Period - VI } & Area & 2.627721 & 0.204066 & -0.04598 \\
\hline & Production & 2.858898 & 0.987032 & 0.059296 \\
\hline & Productivity & 14.14754 & -5.37993 & 1.434513 \\
\hline \multirow{3}{*}{ Period - VII } & Area & 0.000778 & 1.001065 & -1.00262 \\
\hline & Production & -0.00045 & 0.998843 & 1.00156 \\
\hline & Productivity & 0.000443 & -0.99727 & 0.998433 \\
\hline \multirow{4}{*}{ Overall } & Area & -10.3336 & 1.447974 & 1.337308 \\
\hline & Production & 7.136567 & 0.69062 & -0.92357 \\
\hline & Productivity & 7.727142 & 0.747771 & -1.08275 \\
\hline & \multicolumn{4}{|c|}{ Rapeseed \&Mustard } \\
\hline \multirow{3}{*}{ Period - I } & Area & -0.00543 & 0.997662 & -0.99289 \\
\hline & Production & 0.005444 & 1.002344 & 0.99522 \\
\hline & Productivity & -0.0054698 & -1.0071579 & 1.00480286 \\
\hline \multirow{3}{*}{ Period - II } & Area & 5.213295 & 0.072387 & -0.85741 \\
\hline & Production & -55.8022 & 9.826011 & 10.78971 \\
\hline & Productivity & 4.3980924 & -0.6269099 & 0.05811841 \\
\hline \multirow{3}{*}{ Period - III } & Area & 3.780103 & 0.027795 & -0.17642 \\
\hline & Production & -13.9005 & 1.75164 & 5.235113 \\
\hline & Productivity & 2.859646 & -0.338969 & 0.1596087 \\
\hline \multirow{3}{*}{ Period - IV } & Area & 0.008746 & 0.997631 & -0.99786 \\
\hline & Production & -0.00123 & 1.000354 & 1.000083 \\
\hline & Productivity & 0.0023277 & -1.000195 & 0.99969865 \\
\hline \multirow{3}{*}{ Period - V } & Area & 0.006683 & 0.997641 & -0.99692 \\
\hline & Production & -0.00614 & 1.002161 & 0.999343 \\
\hline & Productivity & 0.00612231 & -1.002682 & 1.00058431 \\
\hline \multirow{3}{*}{ Period - VI } & Area & -0.01529 & 1.004772 & -1.00575 \\
\hline & Production & 0.01551 & 0.995185 & 1.00096 \\
\hline & Productivity & -0.01539 & -0.994231 & 0.999026 \\
\hline \multirow{3}{*}{ Period - VII } & Area & -0.00498 & 1.001676 & -1.0021 \\
\hline & Production & 0.004985 & 0.998311 & 1.000441 \\
\hline & Productivity & -0.00490139 & -0.9978066 & 0.99951184 \\
\hline \multirow{3}{*}{ Overall } & Area & 1.382459 & 0.09391 & 0.583595 \\
\hline & Production & -3.60566 & 1.767626 & 1.258601 \\
\hline & Productivity & -0.3306034 & 0.72320207 & 0.08286224 \\
\hline & & Soybean & & \\
\hline & Area & 2.213 & 0.21365 & -1.289563 \\
\hline Period - I & Production & 5.22613 & 0.21356 & -0.56894 \\
\hline & Productivity & 2.231365 & 0.002315 & 0.1289 \\
\hline & Area & -0.26154 & 1.2457 & -0.002148 \\
\hline Period - II & Production & -0.21548 & 2.1367 & -0.00548 \\
\hline & Productivity & -0.31454 & 2.255709 & -0.10055 \\
\hline
\end{tabular}


Sharma Amod

\begin{tabular}{|c|c|c|c|c|}
\hline \multirow{3}{*}{ Period - III } & Area & 2.0103607 & 1.002457 & -0.26154 \\
\hline & Production & 1.002615 & 1.23709 & 0.5648 \\
\hline & Productivity & 0.89546 & 0.75899 & -0.2615 \\
\hline \multirow{3}{*}{ Period - IV } & Area & 1.02985 & 0.23104 & 0.235 \\
\hline & Production & 1.102 & 0.2135 & 0.251 \\
\hline & Productivity & 0.98562 & 0.361 & 0.11124 \\
\hline \multirow{3}{*}{ Period - V } & Area & 1.02356 & 0.3251 & 0.00254 \\
\hline & Production & 0.589613 & -0.26154 & 0.00421 \\
\hline & Productivity & 0.23516 & 0.0125 & 0.0215 \\
\hline \multirow{3}{*}{ Period - VI } & Area & 0.256 & 0.0215 & -0.314537 \\
\hline & Production & 0.03265 & -0.0025 & 0.23546 \\
\hline & Productivity & 0.0213 & 0.0236 & -0.0021 \\
\hline \multirow{3}{*}{ Period - VII } & Area & 0.45231 & 0.2154 & 0.03254 \\
\hline & Production & 0.0215 & 0.3265 & 0.325 \\
\hline & Productivity & 0.03298 & 0.3256 & 0.3251 \\
\hline \multirow{3}{*}{ Overall } & Area & 2.66454E-15 & -0.0001245 & $-2.13365 \mathrm{E}-05$ \\
\hline & Production & $-4.44 \mathrm{E}-16$ & -0.000215 & $-2.64 \mathrm{E}-05$ \\
\hline & Productivity & $2.66 \mathrm{E}-15$ & -0.00022 & $-2.6 \mathrm{E}-05$ \\
\hline \multicolumn{5}{|c|}{ Sunflower } \\
\hline \multirow{3}{*}{ Period - I } & Area & -1.12782 & 1.35E-05 & $1.04 \mathrm{E}-13$ \\
\hline & Production & -1.63011 & 0.007153 & $-1.1 \mathrm{E}-13$ \\
\hline & Productivity & -1.55422 & 0.007977 & $1.04 \mathrm{E}-13$ \\
\hline \multirow{3}{*}{ Period - II } & Area & 772.8118 & $5.15 \mathrm{E}-05$ & $2.4 \mathrm{E}-14$ \\
\hline & Production & -3745.37 & 0.038399 & $-2 \mathrm{E}-14$ \\
\hline & Productivity & -4218 & 0.042153 & $2.4 \mathrm{E}-14$ \\
\hline \multirow{3}{*}{ Period - III } & Area & 0.866384 & 0.388538 & -0.16725 \\
\hline & Production & -2.05854 & 2.462583 & 0.462272 \\
\hline & Productivity & 3.32892 & -3.66144 & 1.59673 \\
\hline \multirow{3}{*}{ Period - IV } & Area & 3.292328 & 0.477969 & -1.14607 \\
\hline & Production & -3.89512 & 1.785524 & 1.59649 \\
\hline & Productivity & 2.941733 & -0.35063 & 0.130749 \\
\hline \multirow{3}{*}{ Period - V } & Area & 0.226945 & 0.963429 & -1.00115 \\
\hline & Production & -0.20282 & 1.026596 & 1.040809 \\
\hline & Productivity & 0.353768 & -0.92166 & 0.899207 \\
\hline \multirow{3}{*}{ Period - VI } & Area & 0.005413 & 1.004198 & -1.01118 \\
\hline & Production & -1.01118 & -0.00481 & 0.995651 \\
\hline & Productivity & 0.006716 & -0.98812 & 0.992427 \\
\hline & Area & -0.19355 & 1.00374 & -0.93796 \\
\hline Period - VII & Production & 0.19283 & 0.99627 & 0.93447 \\
\hline & Productivity & -0.20635 & -1.306614 & 1.07013 \\
\hline & Area & -0.15286 & 0.810372 & -0.5523 \\
\hline Overall & Production & 0.160726 & 1.12027 & 0.806331 \\
\hline & Productivity & 1.089434 & -0.5349 & 0.564896 \\
\hline & & otal Oilseed & & \\
\hline & Area & 0.0180363 & 0.999211745 & -1.00474551 \\
\hline Period - I & Production & -0.0176412 & 1.000651 & 1.005597 \\
\hline & Productivity & 0.0176692 & -0.99489 & 0.9943004 \\
\hline & Area & -0.01362652 & 1.003636722 & -1.0042665 \\
\hline Period - II & Production & 0.022952 & 0.992557 & -0.015781 \\
\hline & Productivity & 0.99932 & -0.994937 & 0.9991956 \\
\hline & Area & -0.01362652 & 1.00363672 & -1.00426656 \\
\hline Period - III & Production & 0.015516 & 0.995847 & 1.000737 \\
\hline & Productivity & -0.01578 & -0.994937 & 0.9991956 \\
\hline & Area & -0.01633689 & 1.004843397 & -1.00639541 \\
\hline Period - IV & Production & 0.016665 & 0.995036 & 1.00162 \\
\hline & Productivity & -0.016796 & -0.993296 & 0.9983263 \\
\hline & Area & 0.003828366 & 1.000066251 & -1.00147656 \\
\hline Period - V & Production & -0.00279 & 0.999681 & 1.001436 \\
\hline & Productivity & 0.0028089 & -0.998175 & 0.9985194 \\
\hline & Area & 0.004922685 & 1.000132318 & -1.00197047 \\
\hline Period - VI & Production & -0.00486 & 0.999847 & 1.001847 \\
\hline & Productivity & 0.0048376 & -0.997984 & 0.9981464 \\
\hline & Area & 0.117347201 & 0.984382687 & -1.00017904 \\
\hline Period - VII & Production & -0.11921 & 1.015865 & 1.016047 \\
\hline & Productivity & 0.1173262 & -0.999821 & 0.9842065 \\
\hline & Area & -0.0008214 & 1.000192865 & -1.00019445 \\
\hline Overall & Production & 0.000878 & 0.999775 & 1.00003 \\
\hline & Productivity & -0.000915 & -0.999685 & 0.9999388 \\
\hline
\end{tabular}

[Table-2] reveals that the ' $c$ ' value in the quadratic functional forms for area, production and productivity were mostly negative impact on different oilseed crops in the country over the periods - I, II, III, IV, V, VI, VII and even overall period. This implies the acceleration of decline in area, production and productivity of different oilseed crops viz; groundnut, rapeseed \& mustard, soybean, sunflower and total oilseed crop. The growth of production and productivity of different oilseed crops were declined over the periods.

[Table-3] reveals that the assumption seemed to be positive, where significant 
compound growth rates were recorded for the growth in area, production and productivity of groundnut, rapeseed and mustard, soybean, sunflower and total oilseed crops during the period under study. Even in case of both soybean and sunflower, it has exhibited a negative impact over the time periods, basically due to decline in area as well as non-adoption of high yielding varieties. The area of different oilseed crops shows negative growth rate due to decline in area over the time periods and shifting of area to other commercial or remunerative crops.
[Table-3] reveals that the co-efficient of variation in area, production and productivity of different oilseed crops are worked out for the periods from 1950-51 to 2012-13 and presented in [Table-3]. It reveals that growing of oilseed crops is moderate risky in India, since it has low coefficient of variation (less than 43.68 per cent). The results of the instability indices in case of area, production and productivity of different oilseed crops in the country are positive which also indicate lower risk for continuing / growing of oilseed crops in future too.

Table-3 Co-efficient of variation (\%) and instability index of area, production and productivity of different oilseed crops

\begin{tabular}{|c|c|c|c|}
\hline Period & Aspects & Co-efficient of Variation & Instability Index \\
\hline \multicolumn{4}{|c|}{ Groundnut } \\
\hline \multirow{3}{*}{ Period-I } & Area & 1.479 & 0.409 \\
\hline & Production & 1.827 & 0.825 \\
\hline & Productivity & 0.928 & 0.798 \\
\hline \multirow{3}{*}{ Period - II } & Area & 0.502 & 0.197 \\
\hline & Production & 1.089 & 1.184 \\
\hline & Productivity & 1.130 & 1.195 \\
\hline \multirow{3}{*}{ Period - III } & Area & 0.260 & 0.067 \\
\hline & Production & 1.309 & 1.661 \\
\hline & Productivity & 1.196 & 1.359 \\
\hline \multirow{3}{*}{ Period - IV } & Area & 0.899 & 0.517 \\
\hline & Production & 2.259 & 3.613 \\
\hline & Productivity & 1.469 & 1.750 \\
\hline \multirow{3}{*}{ Period - V } & Area & 0.751 & 0.070 \\
\hline & Production & 1.364 & 1.779 \\
\hline & Productivity & 15.513 & 1.779 \\
\hline \multirow{3}{*}{ Period - VI } & Area & 0.680 & 0.379 \\
\hline & Production & 2.327 & 5.361 \\
\hline & Productivity & 2.080 & 4.094 \\
\hline \multirow{3}{*}{ Period - VII } & Area & 8.554 & 72.207 \\
\hline & Production & 6.131 & 34.221 \\
\hline & Productivity & 1.067 & 0.0001 \\
\hline \multirow{3}{*}{ Overall } & Area & 0.238 & 0.056 \\
\hline & Production & 0.421 & 0.112 \\
\hline & Productivity & 1.877 & 3.342 \\
\hline \multicolumn{4}{|c|}{ Rapeseed \& Mustard } \\
\hline \multirow{3}{*}{ Period - I } & Area & 1.912 & 0.580 \\
\hline & Production & 6.168 & 1.183 \\
\hline & Productivity & 2.056 & 0.619 \\
\hline \multirow{3}{*}{ Period - II } & Area & 2.640 & 0.064 \\
\hline & Production & 32.061 & 0.144 \\
\hline & Productivity & 0.095 & 0.039 \\
\hline \multirow{3}{*}{ Period - III } & Area & 0.182 & 1.002 \\
\hline & Production & 6.184 & 10.522 \\
\hline & Productivity & 1.654 & 0.730 \\
\hline \multirow{3}{*}{ Period - IV } & Area & 0.146 & 0.377 \\
\hline & Production & -1.191 & 64.951 \\
\hline & Productivity & -1.339 & 0.533 \\
\hline \multirow{3}{*}{ Period - V } & Area & 1.945 & 0.454 \\
\hline & Production & 7.295 & 3.378 \\
\hline & Productivity & 5.250 & 1.353 \\
\hline & Area & 0.723 & 0.202 \\
\hline Period - VI & Production & 0.776 & 10.334 \\
\hline & Productivity & 0.054 & 1.572 \\
\hline & Area & 3.345 & 0.486 \\
\hline Period - VII & Production & 5.704 & 1.480 \\
\hline & Productivity & 2.285 & 1.452 \\
\hline & Area & 2.188 & 0.234 \\
\hline Overall & Production & -3.875 & 2.041 \\
\hline & Productivity & -5.965 & 1.904 \\
\hline & & Soybean & \\
\hline & Area & 1.181 & 0.220 \\
\hline Period - I & Production & 0.802 & 0.571 \\
\hline & Productivity & 1.244 & 0.940 \\
\hline & Area & 0.974 & 0.131 \\
\hline Period - II & Production & 0.271 & 0.072 \\
\hline & Productivity & 1.021 & 0.129 \\
\hline & Area & 10.704 & 23.662 \\
\hline Period - III & Production & 13.410 & 35.596 \\
\hline & Productivity & 10.271 & 42.282 \\
\hline & Area & 4.523 & 1.034 \\
\hline Period - IV & Production & 5.276 & 4.199 \\
\hline & Productivity & 1.361 & 1.745 \\
\hline
\end{tabular}


Sharma Amod

\begin{tabular}{|c|c|c|c|}
\hline \multirow{3}{*}{ Period - V } & Area & 2.796 & 0.299 \\
\hline & Production & 3.569 & 0.722 \\
\hline & Productivity & 1.104 & 0.658 \\
\hline \multirow{3}{*}{ Period - VI } & Area & 1.756 & 0.188 \\
\hline & Production & 15.500 & 236.102 \\
\hline & Productivity & 15.578 & 241.239 \\
\hline \multirow{3}{*}{ Period - VII } & Area & 4.497 & 17.722 \\
\hline & Production & 0.690 & 0.279 \\
\hline & Productivity & 4.135 & 15.173 \\
\hline \multirow{3}{*}{ Overall } & Area & 1.998 & 0.243 \\
\hline & Production & 4.316 & 13.889 \\
\hline & Productivity & 2.912 & 6.578 \\
\hline \multicolumn{4}{|c|}{ Sunflower } \\
\hline \multirow{3}{*}{ Period - I } & Area & 0.755 & 0.218 \\
\hline & Production & 0.221 & 0.048 \\
\hline & Productivity & 0.817 & 0.250 \\
\hline \multirow{3}{*}{ Period - II } & Area & 1.279 & 1.214 \\
\hline & Production & 0.656 & 0.260 \\
\hline & Productivity & 1.520 & 0.934 \\
\hline \multirow{3}{*}{ Period - III } & Area & 4.737 & 22.273 \\
\hline & Production & 8.837 & 75.184 \\
\hline & Productivity & 6.069 & 23.388 \\
\hline \multirow{3}{*}{ Period - IV } & Area & 5.627 & 6.872 \\
\hline & Production & 5.683 & 8.398 \\
\hline & Productivity & 1.806 & 2.243 \\
\hline \multirow{3}{*}{ Period - V } & Area & 1.877 & 2.746 \\
\hline & Production & 2.011 & 3.224 \\
\hline & Productivity & 0.776 & 0.602 \\
\hline \multirow{3}{*}{ Period - VI } & Area & 2.413 & 4.838 \\
\hline & Production & 2.792 & 5.295 \\
\hline & Productivity & 1.337 & 1.486 \\
\hline \multirow{3}{*}{ Period - VII } & Area & 27.671 & 475.430 \\
\hline & Production & 25.142 & 461.267 \\
\hline & Productivity & 3.328 & 0.042 \\
\hline \multirow{3}{*}{ Overall } & Area & 1.529 & 0.888 \\
\hline & Production & 1.664 & 0.956 \\
\hline & Productivity & 0.480 & 0.154 \\
\hline \multicolumn{4}{|c|}{ Total Oilseed } \\
\hline \multirow{3}{*}{ Period - I } & Area & 0.824 & 0.106 \\
\hline & Production & 1.389 & 0.535 \\
\hline & Productivity & 0.826 & 0.495 \\
\hline \multirow{3}{*}{ Period - II } & Area & 0.356 & 0.118 \\
\hline & Production & 0.987 & 0.964 \\
\hline & Productivity & 0.895 & 0.801 \\
\hline & Area & 0.313 & 0.083 \\
\hline Period - III & Production & 1.044 & 1.049 \\
\hline & Productivity & 0.870 & 0.749 \\
\hline & Area & 0.874 & 0.183 \\
\hline Period - IV & Production & 2.209 & 1.963 \\
\hline & Productivity & 1.374 & 1.062 \\
\hline & Area & 0.348 & 0.119 \\
\hline Period - V & Production & 0.967 & 0.507 \\
\hline & Productivity & 0.815 & 0.290 \\
\hline & Area & 0.943 & 0.393 \\
\hline Period - VI & Production & 1.934 & 1.790 \\
\hline & Productivity & 1.322 & 1.206 \\
\hline & Area & 43.675 & 462.675 \\
\hline Period - VII & Production & 0.558 & 0.304 \\
\hline & Productivity & 24.889 & 116.382 \\
\hline & Area & 0.443 & 0.025 \\
\hline Overall & Production & 0.899 & 0.058 \\
\hline & Productivity & 0.488 & 0.023 \\
\hline
\end{tabular}

[Table-4] reveals that on overall time period the productivity of total oilseed crop was found negative with lowest growth rate (-69.276 per cent) as compared to viz; groundnut, soybean, rapeseed \& mustard and sunflower crops, while on the production aspect, it was found to be negative growth rate $(-0.255)$, whereas area was found towards positive impact, which is statistically significant at 1 per cent level and further shows more potentiality to be explore in the days to come. While during the period IV and VI both were found statistically significant at 1 per cent level and during period I and overall time both were found statistically significant either at 1 or 5 per cent level, which is further indication of more potentiality to be explore, while during the remaining periods either it shows positive or negative growth rate on productivity side, which further shows alarming situation and further not to be explore more.

Whereas on groundnut crop area during the period IV, VI and VII were found negative with lowest growth rate (-2.303 per cent) in compare to other periods, which shows due to decline in area; even on production side period III and VI both were found statistically negative impact and on productivity during the period III, it shows negative contribution towards the growth. While on rapeseed \& mustard crop productivity during period IV was found negative with lowest growth rate 
(1.191 per cent), during the period V, it was found to be negative impact toward the development growth. On the soybean crop area during the period VII was found to be lowest growth rate $(-10.423)$ with negative impact, even on the production and productivity side it was found to be negative. While on the sunflower crop productivity during period VII was found to be lowest growth rate (9.547) as compared to other period, even area and production also shows negative towards the development contribution.

\begin{tabular}{|c|c|c|}
\hline Period & Aspects & Compound Growth Rate (\%) \\
\hline \multicolumn{3}{|c|}{ Groundnut } \\
\hline \multirow{3}{*}{ Period - I } & Area & $0.173^{N S}$ \\
\hline & Production & $1.067^{\circ}$ \\
\hline & Productivity & $1.073^{*}$ \\
\hline \multirow{3}{*}{ Period - II } & Area & $4.389^{+1+1+}$ \\
\hline & Production & 5.486 \\
\hline & Productivity & $1.026^{*}$ \\
\hline \multirow{3}{*}{ Period - III } & Area & $0.905^{\circ}$ \\
\hline & Production & $-0.131^{\mathrm{NS}}$ \\
\hline & Productivity & $-1.030 \mathrm{NS}$ \\
\hline \multirow{3}{*}{ Period - IV } & Area & $-0.130 \mathrm{NS}$ \\
\hline & Production & $0.870^{\circ}$ \\
\hline & Productivity & $0.993^{\circ}$ \\
\hline \multirow{3}{*}{ Period - V } & Area & $1.641^{*}$ \\
\hline & Production & $3.756^{* *+}$ \\
\hline & Productivity & $2.077^{\circ}$ \\
\hline \multirow{3}{*}{ Period - VI } & Area & $-2.303^{\mathrm{NS}}$ \\
\hline & Production & $-1.244^{N S}$ \\
\hline & Productivity & $8.406^{\text {t+th }}$ \\
\hline \multirow{3}{*}{ Period - VII } & Area & $-0.965^{\mathrm{NS}}$ \\
\hline & Production & $0.774^{*}$ \\
\hline & Productivity & $1.753^{\circ}$ \\
\hline \multirow{3}{*}{ Overall } & Area & $9.422^{\circ}$ \\
\hline & Production & $3.700^{\circ}$ \\
\hline & Productivity & $2.838^{\circ}$ \\
\hline \multicolumn{3}{|c|}{ Rapeseed \& Mustard } \\
\hline \multirow{3}{*}{ Period - I } & Area & $2.640^{*+1+}$ \\
\hline & Production & $32.061^{\text {t+* }}$ \\
\hline & Productivity & $0.095 \mathrm{NS}$ \\
\hline \multirow{3}{*}{ Period - II } & Area & $0.182^{\mathrm{NS}}$ \\
\hline & Production & $6.184^{*+*}$ \\
\hline & Productivity & $1.654^{*}$ \\
\hline \multirow{3}{*}{ Period - III } & Area & $0.146^{\mathrm{NS}}$ \\
\hline & Production & $-1.191 \mathrm{NS}$ \\
\hline & Productivity & $-1.339 \mathrm{NS}$ \\
\hline \multirow{3}{*}{ Period - IV } & Area & $1.945^{\circ}$ \\
\hline & Production & $7.295^{* * *}$ \\
\hline & Productivity & $5.250^{* *+}$ \\
\hline & Area & $0.723^{*}$ \\
\hline Period -V & Production & $0.776^{*}$ \\
\hline & Productivity & $0.054 \mathrm{NS}$ \\
\hline & Area & $3.345^{* * *}$ \\
\hline Period - VI & Production & $5.704^{* *+}$ \\
\hline & Productivity & $2.285^{* \pm+}$ \\
\hline & Area & $2.188^{\circ}$ \\
\hline Period - VII & Production & $-3.875^{\mathrm{NS}}$ \\
\hline & Productivity & $-5.965^{\mathrm{NS}}$ \\
\hline & Area & $1.912^{\circ}$ \\
\hline Overall & Production & $6.168^{+*+}$ \\
\hline & Productivity & $2.056^{+* t+}$ \\
\hline & Soyk & \\
\hline & Area & $3.434^{\prime \prime \prime}$ \\
\hline Period - I & Production & $0.649 \mathrm{NS}$ \\
\hline & Productivity & $-2.693^{N S}$ \\
\hline & Area & $-2.964^{N S}$ \\
\hline Period - II & Production & $0.084 \mathrm{NS}$ \\
\hline & Productivity & $3.141^{* *}$ \\
\hline & Area & $38.947^{\text {t*t+ }}$ \\
\hline Period - III & Production & $88.572^{\text {t+t }}$ \\
\hline & Productivity & $35.715^{\text {t*t* }}$ \\
\hline & Area & $17.112^{\text {t+t+ }}$ \\
\hline Period - IV & Production & $18.057^{\text {t* }}$ \\
\hline & Productivity & $0.807^{\circ}$ \\
\hline & Area & $10.242^{*+*}$ \\
\hline Period - V & Production & $13.056^{\text {t*t* }}$ \\
\hline & Productivity & $2.553^{\text {"*t+ }}$ \\
\hline
\end{tabular}




\begin{tabular}{|c|c|c|}
\hline \multirow{3}{*}{ Period - VI } & Area & $5.724^{*+*}$ \\
\hline & Production & $10.461^{\text {t*t }}$ \\
\hline & Productivity & $4.480^{+* t+}$ \\
\hline \multirow{3}{*}{ Period - VII } & Area & $-10.423^{\text {NS }}$ \\
\hline & Production & $-1.935^{\mathrm{NS}}$ \\
\hline & Productivity & $9.476^{*}$ \\
\hline \multirow{3}{*}{ Overall } & Area & $13.733^{\text {t*t }}$ \\
\hline & Production & $21.212^{\text {*t* }}$ \\
\hline & Productivity & $6.576^{\text {t*t }}$ \\
\hline \multicolumn{3}{|c|}{ Sunflower } \\
\hline \multirow{3}{*}{ Period - I } & Area & $2.299^{*}$ \\
\hline & Production & $-0.036^{\mathrm{NS}}$ \\
\hline & Productivity & $-2.283^{N S}$ \\
\hline \multirow{3}{*}{ Period - II } & Area & $2.623^{\text {t+t+ }}$ \\
\hline & Production & $-1.303^{\mathrm{NS}}$ \\
\hline & Productivity & $-3.826 \mathrm{NS}$ \\
\hline \multirow{3}{*}{ Period - III } & Area & $0.507 \mathrm{NS}$ \\
\hline & Production & $14.635^{\text {tht }}$ \\
\hline & Productivity & $29.503^{\text {tot }}$ \\
\hline \multirow{3}{*}{ Period - IV } & Area & $25.615^{\text {t*t }}$ \\
\hline & Production & $37.081^{\text {*t* }}$ \\
\hline & Productivity & $-3.478^{N S}$ \\
\hline \multirow{3}{*}{ Period - V } & Area & $-2.969^{N S}$ \\
\hline & Production & $-3.188^{N S}$ \\
\hline & Productivity & $-0.019^{N S}$ \\
\hline \multirow{3}{*}{ Period - VI } & Area & $4.313^{\text {t+t }}$ \\
\hline & Production & $6.101^{*+* t}$ \\
\hline & Productivity & $1.747^{\circ}$ \\
\hline \multirow{3}{*}{ Period - VII } & Area & $47.196^{* * *}$ \\
\hline & Production & $33.829^{\text {t*t }}$ \\
\hline & Productivity & $-9.547 \mathrm{NS}$ \\
\hline \multirow{3}{*}{ Overall } & Area & $5.802^{*+t}$ \\
\hline & Production & $6.959^{*+t}$ \\
\hline & Productivity & $1.066^{*}$ \\
\hline \multicolumn{3}{|c|}{ Total oilseed } \\
\hline \multirow{3}{*}{ Period - I } & Area & $2.501^{*+*+}$ \\
\hline & Production & $4.105^{\text {t*t }}$ \\
\hline & Productivity & $1.557^{*}$ \\
\hline \multirow{3}{*}{ Period - II } & Area & $0.397 \mathrm{NS}$ \\
\hline & Production & $0.287 \mathrm{NS}$ \\
\hline & Productivity & $-0.118^{\mathrm{NS}}$ \\
\hline \multirow{3}{*}{ Period - III } & Area & $0.398 \mathrm{NS}$ \\
\hline & Production & $0.735^{*}$ \\
\hline & Productivity & $0.335^{\mathrm{NS}}$ \\
\hline \multirow{3}{*}{ Period - IV } & Area & $2.441^{\text {t*t+}}$ \\
\hline & Production & $5.454^{* * t}$ \\
\hline & Productivity & $2.952^{\text {t+t }}$ \\
\hline \multirow{3}{*}{ Period - V } & Area & $0.166^{\mathrm{NS}}$ \\
\hline & Production & $2.250^{*}$ \\
\hline & Productivity & $2.073^{*}$ \\
\hline & Area & $2.450^{+*+1}$ \\
\hline Period - VI & Production & $5.135^{\text {t*t }}$ \\
\hline & Productivity & $2.620^{\text {t*t }}$ \\
\hline & Area & $224.732^{4 * t+}$ \\
\hline Period - VII & Production & $-0.255^{\mathrm{NS}}$ \\
\hline & Productivity & $-69.276^{\mathrm{NS}}$ \\
\hline & Area & $1.525^{*}$ \\
\hline Overall & Production & $3.045^{\text {t*t }}$ \\
\hline & Productivity & $1.498^{*}$ \\
\hline
\end{tabular}

( ${ }^{*}$ Significant at 1 per cent; * Significant at 5 per cent; * Significant at 50 per cent; NS - Non Significant)

[Table-5] reveals that the relative effect of contributions of area, productivity and their interactions on increased production of different oilseed crops in the country for the periods from 1950-51 to 1959-60; 1960-61 to 1969-70; 1970-71 to 197980; $1980-81$ to $1989-90 ; 1990-91$ to $1999-00 ; 2000-01$ to $2009-10 ; 2010-11$ to 2012-13 and 1950-51 to 2012-13 have been estimated. It reveals that the production of the different oilseed crops in India has exhibited an increasing trend except soybean and sunflower during the periods II, IV and VII. This increase in production was due to increase in area as well as interactions of area and productivity of oilseed crops during the period under review. Further similar type of trend results were reported by the Das \& Sharma (2012)[5]; Sharma (2012)[8]; Sharma (2013b)[10]; Sharma (2015c)[14]; Sharma \& Kalta (2008)[16].

\section{Conclusion and Policy Implication}

The above discussion highlighted the fact that the growth of area, production and productivity for the different oilseed crops in the Country were found to be positive as well as statistically significant too. The coefficient of variation for almost all the crops were less than 43.68 per cent thereby indicating moderate risky for the cultivation of oilseed crops during various time period in the Country. This was also indicated by the lower value of instability indices. Keeping the area as constant, the productivity can be further increased by taking appropriate production technologies or introducing high yield varieties to overcome the problems of population pressure in the coming days may to solve up to maximum extend. 


\begin{tabular}{|c|c|c|c|c|c|}
\hline \multicolumn{6}{|c|}{ Table-5 Effect of change in area, productivity and their interactions on production of oilseed crops } \\
\hline Crops & $\begin{array}{l}\text { Aspects } \\
\text { (Period) }\end{array}$ & $\begin{array}{c}\text { Differential } \\
\text { production }(\Delta \mathrm{P})\end{array}$ & $\begin{array}{l}\text { Area effect } \\
\left(Y_{0} \triangle A\right)\end{array}$ & $\begin{array}{c}\text { Productivity effect } \\
\left(\mathrm{A}_{0} \Delta \mathrm{Y}\right)\end{array}$ & $\begin{array}{c}\text { Interaction } \\
(\Delta \mathrm{A} \Delta \mathrm{Y})\end{array}$ \\
\hline \multirow{8}{*}{ Groundnut } & $1950-51$ to $1959-60$ & 1511250 & -300830 & -130650 & 1079770 \\
\hline & $1960-61$ to $1969-70$ & 499150 & -161500 & -16750 & 320900 \\
\hline & $1970-71$ to $1979-80$ & -133440 & -212570 & 4640 & -341370 \\
\hline & $1980-81$ to $1989-90$ & 1405760 & 1619200 & 370540 & 3095500 \\
\hline & $1990-91$ to $1999-00$ & -130176 & -1146780 & 198720 & -2249820 \\
\hline & $2000-01$ to $2009-10$ & -105516 & 91840 & -15120 & -978440 \\
\hline & $2010-11$ to $2012-13$ & 1488632 & 434350 & 85702 & 2008684 \\
\hline & $1950-51$ to $2012-13$ & 3379000 & 1149440 & 1116160 & 5644600 \\
\hline \multirow{8}{*}{$\begin{array}{l}\text { Rapeseed } \\
\text { \& Mustard }\end{array}$} & $1950-51$ to $1959-60$ & 309120 & -6210 & -2520 & 300390 \\
\hline & $1960-61$ to $1969-70$ & 135430 & 74880 & 7540 & 217850 \\
\hline & $1970-71$ to $1979-80$ & 89100 & -607560 & -27450 & -545910 \\
\hline & $1980-81$ to $1989-90$ & 481600 & 1113810 & 233060 & 1828470 \\
\hline & $1990-91$ to $1999-00$ & 226000 & 323680 & 14000 & 563680 \\
\hline & $2000-01$ to $2009-10$ & 1037850 & 1106560 & 274170 & 2418580 \\
\hline & $2010-11$ to $2012-13$ & 339552 & -885360 & -39168 & -584976 \\
\hline & $1950-51$ to $2012-13$ & 1739904 & 1397250 & 3191400 & 6328554 \\
\hline \multirow{8}{*}{ Soybean } & $1950-51$ to $1959-60$ & 666.6667 & -556.25 & -185.417 & -75 \\
\hline & $1960-61$ to $1969-70$ & 780900 & 17750 & 7295250 & 8093900 \\
\hline & $1970-71$ to $1979-80$ & 45619.05 & 9760 & 222621 & 278000 \\
\hline & $1980-81$ to $1989-90$ & 1182951 & 50711.11 & 136338.1 & 1370000 \\
\hline & $1990-91$ to $1999-00$ & 3717188 & 313955 & 448857.5 & 4480000 \\
\hline & $2000-01$ to $2009-10$ & 2722243 & 1291757 & 665999.6 & 4680000 \\
\hline & $2010-11$ to $2012-13$ & -250151 & 2414950 & -496933 & -483493 \\
\hline & $1950-51$ to $2012-13$ & 727809.5 & 31369 & 11415328 & 12174507 \\
\hline \multirow{8}{*}{ Sunflower } & $1950-51$ to $1959-60$ & 15000 & -11486.154 & -3445.846 & 68 \\
\hline & $1960-61$ to $1969-70$ & 10885.5349 & -12332.845 & -2485.69 & -3933 \\
\hline & $1970-71$ to $1979-80$ & -4000 & 54280 & -27140 & 23140 \\
\hline & $1980-81$ to $1989-90$ & 593850 & -3120 & -27820 & 562910 \\
\hline & $1990-91$ to $1999-00$ & -181900 & 4890 & -1020 & -178030 \\
\hline & $2000-01$ to $2009-10$ & 246820 & -27820 & -10660 & 208340 \\
\hline & $2010-11$ to $2012-13$ & 730800 & -114300 & -133350 & 483150 \\
\hline & $1950-51$ to $2012-13$ & 925000 & 6900 & 127650 & 1059550 \\
\hline \multirow{8}{*}{$\begin{array}{c}\text { Total } \\
\text { Oilseed } \\
\text { Crops }\end{array}$} & $1950-51$ to $1959-60$ & 1548820 & -118030 & -35420 & 1395370 \\
\hline & $1960-61$ to $1969-70$ & 527280 & 206550 & 15600 & 749430 \\
\hline & $1970-71$ to $1979-80$ & 173700 & -104832 & -18900 & -893520 \\
\hline & $1980-81$ to $1989-90$ & 2766400 & 3696000 & 1092000 & 7554400 \\
\hline & 1990-91 to 1999-00 & 100230 & 1980300 & 10660 & 2091190 \\
\hline & $2000-01$ to $2009-10$ & 2583900 & 3392730 & 475310 & 6451940 \\
\hline & $2010-11$ to $2012-13$ & $2.97 \mathrm{E}+08$ & -2815017 & $-2.70 E+08$ & -14286 \\
\hline & $1950-51$ to $2012-13$ & 8441550 & 6577490 & 10758150 & 25777190 \\
\hline
\end{tabular}

There existed severe ups and down in the growth process repealing the smoothness of growth in the area, production and yield of different oilseed crops in the country. In view of the above findings following suggestions are recommended for suitable policy formation in the days to come.

Yield growth rate is skimpy because of poor availability of high yield variety of oil seed crops, wide spread infestation by insects, pests and disease, destruction of crops by animals in the field, inadequate and irregular rainfall / water supply to the oilseed crops etc. So, to increase the yield growth rate, steps should be taken to overcome the mentioned difficulties face by the cultivators.

A rising trend in the growth featured by high degree of variability is a sign of vulnerability in the growth process. Wide spread ups and downs in the area, production and yield of oilseed crops, shatters the rational expectations of the cultivators and lead to many disruptive consequences.

Thus, there is need for proper policies and programmes to concentrate on increasing the production and yield of major oilseed crops by introducing HYVs and by increasing area under cultivation to include non-traditional areas and encourage the farmers to use appropriate amount of inputs viz.; fertilizers, improved seeds, pesticides and irrigation water etc.

\section{Application of research}

The present research article will be useful to the researchers, teachers, policy makers, planner, extension workers, government institution, industrial agencies, processing units, NGO's etc to understand the current trends of oilseed crops production and making future effective strategies in the days to come.
Research Category: Field of oilseed crops

\section{Abbreviations}

CV: Coefficient of Variation

CGR: Compound Growth Rate

I: Instability Index

Acknowledgement / Funding: Author thankful to Nagaland University, Medziphema Campus, Dimapur, Nagaland, 797106

\section{*Principle Investigator: Dr Amod Sharma}

University: Nagaland University, Medziphema Campus, Dimapur, Nagaland, 797106

Research project name or number: NIL

Author Contributions: Sole author

Author statement: Author read, reviewed, agree and approved the final manuscript

\section{Conflict of Interest: None declared}

Ethical approval: This article does not contain any studies with human participants or animals performed by any of the authors. 


\section{References}

[1] Anonymous (2011) Agricultural Statistics at a glance. Directorate of Economics and Statistics. Department of Agriculture and Cooperation. Ministry of Agriculture, New Delhi.

[2] Anonymous (2013a) http://agricoop.nic.in/eands. Accessed on 23rd March 2013.

[3] Anonymous (2013b) http://dacnet.nic.in/eands. Accessed on 24th March 2013.

[4] Cavery R. (1991) Agricultural Situation in India. 46(5), 319-321.

[5] Das, Kandarpa. Kumar and Sharma Amod (2012) Progressive Agriculture, 12(2), 392-395.

[6] Dhakre D. S. and Sharma Amod (2010a) Environment \& Ecology, 28(1A), 270-272.

[7] Dhakre D. S. and Sharma Amod (2010b) Agriculture Science Digest., 30(2), 142-144.

[8] Sharma Amod (2012) Economic Affair. 57(3), 259-276.

[9] Sharma Amod (2013a) Agriculture Science Digest., 33(1), 60-62.

[10] Sharma Amod (2013b) Economic Affairs. 58(1), 57-68.

[11] Sharma Amod (2014) Economic Affairs. 59(4), 569-578.

[12] Sharma Amod (2015a) Agricultural Science Digest., 36(1), 7-12.

[13] Sharma Amod (2015b) International Journal of Agriculture Innovations \& Research, 4(3), 509-511.

[14] Sharma Amod (2015c) Journal of Spices and Aromatic Crops. 24(2), 112118.

[15] Sharma Amod and Kalita D.C. (2004) Nagaland University Research Journal, 2, 31-37.

[16] Sharma Amod and Kalita D.C. (2008) Agricultural Situation in India, LXV(7), 477-488.

[17] Yadav Mukesh Kumar, Sharma Amod, Rajbhar Arun Kumar and Kalai, Kankabati (2017) Plant Archiver, 17(2), 1481-1491 